\title{
Optical Transfer Functions of Kerr Nonlinear Cavities and Interferometers
}

\author{
Henning Rehbein, ${ }^{1}$ Jan Harms, ${ }^{1}$ Roman Schnabel, ${ }^{1}$ and Karsten Danzmann ${ }^{1}$ \\ ${ }^{1}$ Institut für Atom- und Molekülphysik, Universität Hannover and Max-Planck-Institut für Gravitationsphysik \\ (Albert-Einstein-Institut), Callinstrasse 38, 30167 Hannover, Germany
}

(Received 20 May 2005; published 1 November 2005)

\begin{abstract}
We present the optical transfer functions for third-order nonlinear cavities that involve an optical carrier frequency and its modulation sideband fields. Our approach is based on linearized transformations and provides a convenient tool to calculate squeezed light sources as well as complex interferometer topologies, containing subsystems that involve intensity dependent phase shifts, i.e., optical Kerr media. As the result we present the noise spectral density of a Michelson interferometer with Kerr nonlinear arm cavities and resonant sideband extraction and find that quantum noise can be squeezed by arbitrary amounts even outside the cavity linewidth. Such a system might apply for future gravitational wave detectors or simply for a continuous wave source of squeezed states.
\end{abstract}

DOI: 10.1103/PhysRevLett.95.193001

The input and output of damped quantum optical systems have been successfully described by quantum Langevin equations. This approach led to the first correct description of traveling wave squeezed light [1]. However, this approach is not easy to deal with due to its nonlinear operator nature. Especially the description of complex optical systems, like advanced laser interferometers that comprise a detuned resonant sideband extraction (RSE) cavity [2] in combination with radiation pressure and nonlinear media, is a nontrivial task. Fortunately, for most practical purposes the quantum noise can be assumed to be small compared to the field's expectation value and the problem can be linearized. Following this line, in Refs. [3,4] the Michelson interferometer with Kerr media placed into the arm cavities has been analyzed. It was shown that Kerr media inside the arms can cancel radiation pressure noise at some sideband frequencies.

The interest in nonclassical techniques has been increasing considerably during recent years. This is partly due to the fact that quantum noise will be one of the major noise sources in second-generation interferometric gravitational wave (GW) detectors (e.g., Advanced LIGO [5]). It is therefore very likely that third-generation detectors will exploit quantum correlations, i.e., quantum-nondemolition techniques [6,7] and nonlinear optics might become one of the standard tools.

In this Letter we reinvestigate the Kerr medium inside the arm cavities of a Michelson interferometer and present signal and noise transfer functions. For the first time, to our best knowledge, we report the noise spectral density of a Kerr-effect enhanced Michelson interferometer with RSE. We apply a theoretical approach which can easily be extended to even more complex optical systems. Our formalism is based on linearized transformations of the quadrature fields within two-photon quantum optics [8] which has previously been used to describe ponderomotive squeezing [6] and the optical spring effect [5]. In this formalism the transfer functions map quadrature amplitudes which act at positive modulation frequencies $\Omega$
PACS numbers: 42.50.Dv, 03.65.Ta

around an optical carrier frequency $\omega_{0}$. It is well-known that cavities containing a $\chi^{(3)}$ nonlinear crystal (Fig. 1) can provide an improved response to phase signals and quantum noise can become (Kerr) squeezed [9]. The nonlinear relation between the input intensity and the intracavity intensity reads

$$
I_{\mathrm{cav}}=\frac{\tau_{1}^{2}}{1+\rho_{1}^{2} \rho_{2}^{2}-2 \rho_{1} \rho_{2} \cos \left(2 \phi_{\mathrm{det}}+2 \theta I_{\mathrm{cav}}\right)} I_{\mathrm{in}},
$$

where $\phi_{\operatorname{det}}$ denotes the cavity detuning and $\theta$ accounts for the $\chi^{(3)}$ nonlinearity; for $\theta=0$ we obtain the intracavity intensity of a linear cavity. In Fig. 2, the phase shifts of light reflected from a Kerr cavity and the intracavity intensities are shown as a function of the cavity detuning. There is a critical choice of parameters where the phase response to a small change of the cavity detuning is infinite for one specific value of the detuning and where the cavity does not assume a multistable state. This critical state is considered to be very promising in view of enhancing the sensitivity for detecting signals induced by a cavity length change.

For a rigorous treatment we start from the system's Kerr Hamiltonian in the interaction picture

$$
\hat{H}=\frac{\hbar}{2} \chi\left(\hat{a}^{\dagger}\right)^{2} \hat{a}^{2},
$$

where $\hat{a}$ is the field annihilation operator acting at a par-

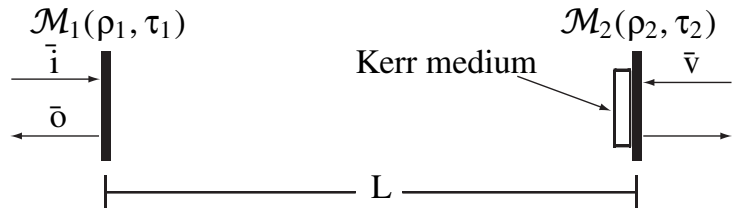

FIG. 1. Kerr nonlinear cavity with laser and vacuum inputs described by quadrature amplitude vectors. Mirror amplitude reflectance and transmittance are denoted by $\rho$ and $\tau$, respectively. $L$ is the macroscopic length of the cavity. 

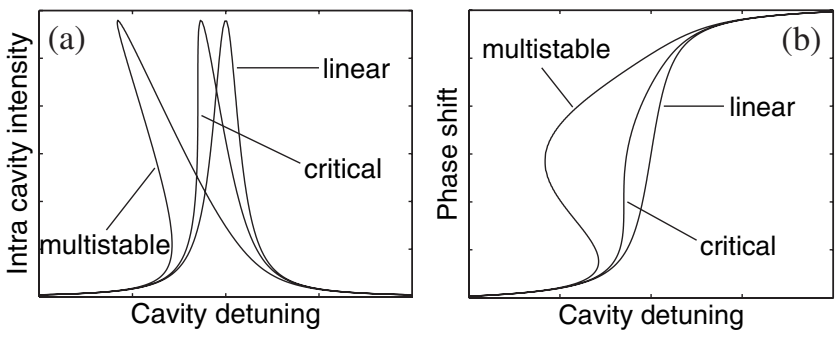

FIG. 2. (a) Intracavity powers and (b) phase shifts of reflected light for three cavities of different values for the nonlinear susceptibility of the Kerr medium. The critical state is characterized by possessing infinite slope at some single value for the detuning.

ticular frequency, and so the Hamilton operator just involves one frequency. The quantity $\chi$ is proportional to the nonlinear susceptibility of the Kerr medium used. Denoting the interaction time by $\tau$, the equation of motion becomes

$$
\frac{\mathrm{d} \hat{a}}{\mathrm{~d} \tau}=-\mathrm{i} \chi \hat{a}^{\dagger} \hat{a} \cdot \hat{a}=-\mathrm{i} \chi \hat{n} \cdot \hat{a} .
$$

which is solved easily by making use of the fact that the photon number is conserved during the interaction with the Kerr medium:

$$
\hat{a}(\tau)=e^{-\mathrm{i} \chi \tau \hat{n}(0)} \cdot \hat{a}(0) .
$$

At first, we procure the usual linearized transformation rule that applies for intense optical fields weakly interacting with a Kerr medium. Therefore, the annihilation operator is decomposed into its (real) expectation value and a noise amplitude operator $\hat{a}(\tau)=\Lambda+\hat{v}(\tau)$. Keeping just first order terms of $\hat{v}$ we obtain from Eq. (4)

$$
\hat{v}(\tau)=e^{-\mathrm{i} \chi \tau \Lambda^{2}}\left[\hat{v}-\mathrm{i} \chi \tau \Lambda^{2} \cdot\left(\hat{v}+\hat{v}^{\dagger}\right)\right]
$$

The linearized solution has to be evaluated for two sideband frequencies $\omega_{0} \pm \Omega$ in order to obtain a field representation in terms of the quadrature amplitudes $\hat{v}_{1,2}(\tau)$ of the two-photon formalism [8]. The noise power spectral density of a phase sensitive photo detection $[7,10]$ can be derived from the components of the Fourier-transformed covariance matrix for the amplitude and phase quadratures of the light field. To ease notation, we define the quadrature amplitude vector for small modulation frequencies $\Omega \ll \omega_{0}$

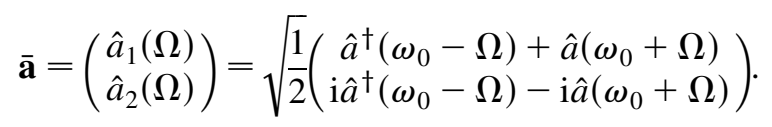

We now define the Kerr coupling constant $\mathcal{K}_{\text {op }} \equiv 2 \chi \tau \Lambda^{2}$ such that the respective linearized transformation of the quadrature noise fields assumes the form

$$
\left(\begin{array}{l}
\hat{v}_{1}(\tau) \\
\hat{v}_{2}(\tau)
\end{array}\right)=\mathbf{D}\left[-\mathcal{K}_{\mathrm{op}} / 2\right] \underbrace{\left(\begin{array}{cc}
1 & 0 \\
-\mathcal{K}_{\mathrm{op}} & 1
\end{array}\right)}_{\equiv \mathbf{K}}\left(\begin{array}{l}
\hat{v}_{1} \\
\hat{v}_{2}
\end{array}\right),
$$

where $\mathbf{D}$ denotes the $2 \times 2$ rotation matrix. One sees that the linearized Kerr transformation consists of a rotation in addition to a conversion of amplitude quadrature fluctuations into phase quadrature fluctuations.

In the above quadrature notation the effect of a Kerr medium is described by the Kerr coupling constant $\mathcal{K}_{\mathrm{op}}$. In case of a Kerr nonlinear crystal (electro-optical Kerr effect) the coupling constant is frequency independent over a broad spectrum and reads

$$
\mathcal{K}_{\text {op }} \equiv 2 \chi \tau \Lambda^{2}=\left(1+\rho_{2}^{2}\right) \frac{n_{2} \omega_{0} L_{\mathrm{op}} I}{c} \equiv \theta I,
$$

where $n_{2} \propto \chi$ is the nonlinear refractive index with units $\mathrm{m}^{2} / \mathrm{W}, L_{\mathrm{op}}$ is the length of the Kerr medium and $I$ denotes the light intensity inside the Kerr medium. The factor $(1+$ $\rho_{2}^{2}$ ) already anticipates the fact that the Kerr medium is placed inside a cavity and consequently the intensity is a sum of the intensities of two counter-propagating beams. The nonlinearities required for our purposes are experimentally feasible (cf. [11]).

Note that radiation pressure as well as thermal expansion due to light absorption and a temperature dependent refractive index of substrates also result in intensity dependent phase shifts. In analogy to Eq. (8) these effects may be described by appropriate coupling parameters $\mathcal{K}_{\mathrm{rp}}$ and $\mathcal{K}_{\text {th }}$, respectively. The radiation pressure effect was indeed studied in great detail in Refs. [6,5] where the coupling parameter $\mathcal{K}_{\text {rp }}$ was introduced. Both radiation pressure and photo-thermally induced nonlinear phase shifts generally decrease with increasing frequency on scales which are smaller than the detection bandwidth.

We will now derive the signal and quantum noise transfer functions of the Kerr nonlinear cavity. According to Fig. 1 a (detuned) cavity is pumped by a coherent input field of frequency $\omega_{0}$ through mirror $\mathcal{M}_{1}$, denoted by $\overline{\mathbf{i}}$. Propagation of the field from the first to the second mirror $\mathcal{M}_{2}$ is performed by the transfer matrix

$$
\mathbf{P}_{L}=e^{\mathrm{i} \Phi} \mathbf{D}[-\phi] \text {. }
$$

To take the sidebands into account, the (complex) phase with $\Phi=\Omega L / c$ is required. The rotation angle $\phi$ corresponds to the phase shift of the carrier light. Here, two different contributions have to be considered:

$$
\phi=\phi_{\text {det }}+\frac{\mathcal{K}_{\mathrm{op}}}{2}
$$

where $\phi_{\text {det }}$ denotes the detuning of the cavity and $\mathcal{K}_{\mathrm{op}} / 2$ is the classical phase shift of the light due to the Kerr medium. The phase of the incoming beam is chosen such that the carrier field is rotated into the amplitude quadrature of our reference system when impinging on $\mathcal{M}_{2}$ (then $\Lambda$ is real). Here, at $\mathcal{M}_{2}$ we place the Kerr medium, which is then described by Eq. (7), and the round trip matrix for the whole cavity reads

$$
\mathbf{R}=\rho_{2} \mathbf{P}_{L} \cdot \mathbf{K} \cdot \mathbf{P}_{L}
$$


The noise transfer matrix of the (detuned) nonlinear cavity is given by the following reflection transfer matrix

$$
\mathbf{C}=\frac{1}{\mathbb{1}-\rho_{1} \mathbf{R}} \cdot\left[\mathbf{R}-\rho_{1} \cdot \mathbb{1}\right]
$$

We now consider a classical signal that induces a motion of mirror $\mathcal{M}_{2}$ giving rise to a phase modulation of the reflected fields. The signal transfer matrix from $\mathcal{M}_{2}$ to the output port $\overline{\mathbf{0}}$ is given by

$$
\mathbf{S}=\frac{\tau_{1}}{\mathbb{1}-\rho_{1} \mathbf{R}} \cdot \mathbf{P}_{L}
$$

Vacuum fields attributed to losses inside the cavity will give a third contribution to the overall cavity output field. This is described by the vacuum field $\overline{\mathbf{v}}$ impinging from outside the cavity on $\mathcal{M}_{2}$ (Fig. 1). The loss transfer matrix is found to be $\tau_{2} \mathbf{S}$. Eventually, the input-output relation for a single cavity can be cast into the following form:

$$
\overline{\mathbf{o}}=\mathbf{C} \cdot \overline{\mathbf{i}}+\mathbf{S} \cdot\left[\Lambda \frac{\omega_{0}}{c}\left(\begin{array}{c}
0 \\
2 x(\Omega)
\end{array}\right)+\tau_{2} \overline{\mathbf{v}}\right],
$$

where $x(\Omega)$ is the amplitude of relative displacements between the two cavity mirrors (e.g., a gravitational wave of amplitude $h(\Omega)$ leads to $\left.x(\Omega)= \pm \frac{1}{2} \operatorname{Lh}(\Omega)[6]\right)$.

Now two cavities described by Eq. (14) may be combined to form a Michelson interferometer operating close to a dark fringe. Then, keeping the value for the laser power in each arm, its antisymmetric signal mode is described by the signal transfer matrix given above multiplied by $\sqrt{2}$. The transfer matrix $\mathbf{C}$ and the adjusted signal transfer matrix $\mathbf{S}$ directly translate into the spectral noise density of the interferometer for a measured quadrature $\hat{o}_{\zeta}=$ $\hat{o}_{1} \cos \zeta+\hat{o}_{2} \sin \zeta$, where $\zeta$ is the homodyne angle; see, for example, Eq. 7 in Ref. [7].

In Figs. 3 and 4 we plot transfer functions and spectral noise densities of linear and different Kerr nonlinear Michelson interferometers. In all cases the curves represent the optimum choice of quadrature angle and arm cavity detuning. In the linear case tuned arm cavities and the phase quadrature provide the lowest spectral noise density. In the nonlinear case we initially chose to minimize spectral noise density at zero frequency and found the interferometer to perform best with detuned arm cavities in the critical state when observing the amplitude quadrature. Figure 3 shows that for $\Omega=0$ a complete cancellation of noise can be observed for the Kerr nonlinear interferometer (solid line). Note that Fig. 2 implies an amplification of the phase quadrature signal transfer function. Indeed, this can be accomplished by an appropriate choice of the detection angle. However, it turns out that the noise transfer function is amplified as well and the net gain of sensitivity is less compared to the detection of the amplitude quadrature. Up to this point our analysis of the Kerreffect enhanced interferometer can be summarized as follows. The quantum measurement noise (shot-noise) transfer function of a Michelson interferometer with detuned

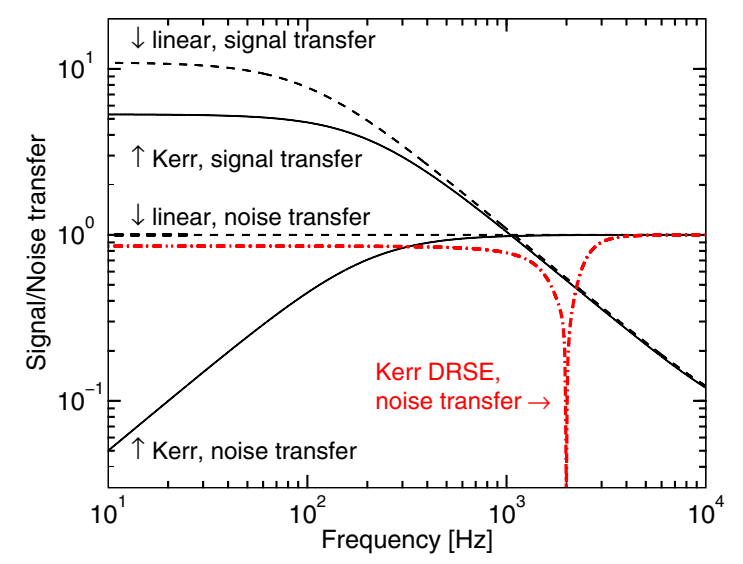

FIG. 3 (color online). Transfer functions of Michelson interferometers with infinite mirror masses; comparison of tuned linear arm cavities with nonlinear Kerr arm cavities close to the critical state. The peaked curve results from additional DRSE. Parameters: $\omega_{0}=1.77 \times 10^{15} \mathrm{~Hz}, \quad \gamma_{\mathrm{arm}} \simeq 2 \pi \times$ $100 \mathrm{~Hz}, \quad L=4 \mathrm{~km}, \quad I=10 \mathrm{~kW} / \mathrm{cm}^{2}$. Without DRSE cavity: $\quad n_{2} L_{\text {op }}=6.5210^{-17} \mathrm{~m}^{3} / \mathrm{W}, \quad \mathcal{K}_{\text {op }}=0.0757, \quad \phi=$ 0.0359. With DRSE cavity: $\tilde{\rho}_{1}=0.546, \tilde{\rho}_{2}=0.9, \tilde{L}=4 \mathrm{~km}$, $n_{2} L_{\text {op }}=1.55 \times 10^{-16} \mathrm{~m}^{3} / \mathrm{W}, \mathcal{K}_{\text {op }}=0.18, \phi=-1.5573$.

Kerr arm cavities can be reduced significantly for sideband frequencies smaller than the half linewidth of the (single ended) arm cavity

$$
\gamma_{\mathrm{arm}}=\tau_{1}^{2} c /(4 L) .
$$

At the same time the signal transfer is also reduced, but just by a small value. The result is an improved noise spectral density. Unfortunately the improved frequency band is also restricted by a lower bound set by radiation pressure noise (backaction noise) since its transfer function equals the signal transfer function. An overall reduction of the noise spectral density for an amplitude quadrature measurement

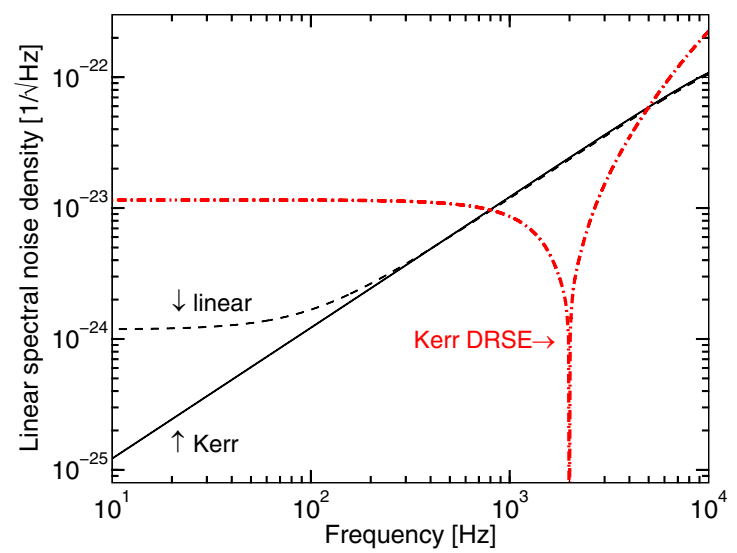

FIG. 4 (color online). Linear spectral noise densities of GW interferometers according to Fig. 3. For a nonlinear Michelson without DRSE the spectral noise density can be made zero at zero frequency assuming no radiation pressure effect. With DRSE infinite improvement can be achieved at some sideband frequency where the linear interferometer may be shot-noise limited. 
is therefore restricted to shot-noise limited frequencies within the half linewidth of the cavity.

An interesting question arises if the effect of noise reduction can be shifted to higher frequencies well above the cavities' half linewidth where radiation pressure noise (as well as technical laser noise) are negligible. The answer to this question is "yes," if an appropriate extended RSE topology is applied. One finds that the formula describing a Michelson interferometer with detuned arm cavities presented here, holds also for an interferometer with tuned arm cavities and RSE having a single mirror at the interferometer's dark port, as analyzed by Buonanno and Chen [5]. Simply adjusting the parameter $\Phi$ (cf. Table II in Ref. [7]) $\Phi \rightarrow \arctan \left(\Omega / \gamma_{\text {arm }}\right)$, yields the well-known RSE formulas. However, in this topology the upper or the lower signal sideband is extracted from low linewidth arm cavities. In our case both sidebands need to be extracted to preserve the nonclassical noise suppression and another complex phase factor has to be added to the round trip matrix in Eq. (11). From previous investigations [5] it is clear that the appropriate phase factor can experimentally be realized by a newly established doubly resonant sideband extraction (DRSE) cavity at the dark port instead of a single mirror, as shown in Fig. 5. The DRSE cavity has to be tuned on resonance with respect to the carrier field, since in the (D)RSE topology the Kerr arm cavities are also resonating. Note that the latter fact gives another advantage because the arm cavity's power buildup is higher. By adjusting the parameters of the DRSE cavity the Kerr noise reduction can be shifted to arbitrary frequencies. The peaked curves in Figs. 3 and 4 show the nonclassical improvement due to the Kerr nonlinear DRSE topology. Again noise reduction by arbitrary amounts is possible, but now for sideband frequencies outside the half linewidth of the arm cavity with a resonance at

$$
\Omega_{\mathrm{res}}=\sqrt{\gamma_{\mathrm{arm}}\left[\gamma_{\mathrm{arm}}+\frac{c}{\tilde{L} \tilde{\tau}_{1}^{2} \tilde{\rho}_{2}}\left(\tilde{\rho}_{2}+\tilde{\rho}_{1}\right)\left(1+\tilde{\rho}_{1} \tilde{\rho}_{2}\right)\right]}
$$

where the tilde refers to the DRSE cavity.

The Kerr DRSE interferometer proposed here might find applications in large scale GW detectors as well as in continuous wave (cw) squeezed light sources. Although the Kerr effect has appeared to be a technically simple approach to generate squeezed states, only marginal degrees of cw Kerr squeezing has been demonstrated. So far this is due to the low values of third-order nonlinearities in crystals. To achieve high intensities, small linewidth cavities have to be used. Without DRSE, squeezing is only expected within the half linewidth but then typically buried by huge classical noise. Only in the pulsed regime has Kerr squeezing of up to $7 \mathrm{~dB}$ been demonstrated [12]. Soliton pulses propagating through fibers provide high intensities as well as long interaction length without the need for cavities. Kerr squeezing in atoms was also successful to some extent due to the orders of magnitude higher nonlinearity [13]. We believe that our proposed DRSE topol-

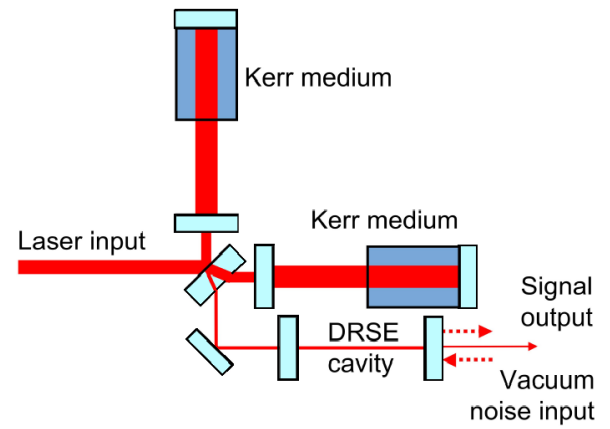

FIG. 5 (color online). Kerr nonlinear Michelson interferometer operated with a signal port close to a dark fringe. The inputoutput relation of each arm is given by Eq. (14). We consider two different cases, without and with additional optics for DRSE in the dark port.

ogy will solve the driving noise problem in cw Kerr squeezing experiments. The squeezed light source is operated with tuned, small linewidth cavities providing the required power built up, but squeezing will be generated at higher frequencies at which technical laser noise is rejected.

We thank Y. Chen for many helpful discussions concerning the construction of a program which simulates the Kerr interferometer and for his suggestions which influenced the design of our analysis.

[1] M. J. Collett and C. W. Gardiner, Phys. Rev. A 30, 1386 (1984); C. W. Gardiner and M. J. Collett, Phys. Rev. A 31, 3761 (1985).

[2] J. Mizuno, K. A. Strain, P. G. Nelson, J. M. Chen, R. Schilling, A. Rüdiger, W. Winkler, and K. Danzmann, Phys. Lett. A 175, 273 (1993).

[3] R. S. Bondurant and J. H. Shapiro, Phys. Rev. D 30, 2548 (1984).

[4] A. F. Pace, M. J. Collett, and D. F. Walls, Phys. Rev. A 47, 3173 (1993).

[5] A. Buonanno and Y. Chen, Phys. Rev. D 65, 042001 (2002).

[6] H. J. Kimble, Y. Levin, A. B. Matsko, K. S. Thorne, and S. P. Vyatchanin, Phys. Rev. D 65, 022002 (2002).

[7] J. Harms, Y. Chen, S. Chelkowski, A. Franzen, H. Vahlbruch, K. Danzmann, and R. Schnabel, Phys. Rev. D 68, 042001 (2003).

[8] C. M. Caves and B. L. Schumaker, Phys. Rev. A 31, 3068 (1985).

[9] M. Kitagawa and Y. Yamamoto, Phys. Rev. A 34, 3974 (1986).

[10] A. Buonanno, Y. Chen, and N. Mavalvala, Phys. Rev. D 67, 122005 (2003).

[11] D. A. B. Miller, D. S. Chemla, D. J. Eilenberger, P. W. Smith, A.C. Gossard, and W. Wiegmann, Phys. Rev. A 36, 5271 (1987).

[12] S. Schmitt, J. Ficker, M. Wolff, F. Knig, A. Sizmann, and G. Leuchs, Phys. Rev. Lett. 81, 2446 (1998).

[13] A. Lambrecht, T. Coudreau, A. M. Steinberg, and G. Giacobino, Europhys. Lett. 36, 93 (1996). 